suggest that the procedure might be applicable in the prevention of recurrent stenosis.

Original article Lekston A et al. (2008) Angiographic and intravascular ultrasound assessment of immediate and 9-month efficacy of percutaneous transluminal renal artery balloon angioplasty with subsequent brachytherapy in patients with renovascular hypertension. Kidney Blood Press Res 31: 291-298

\section{Meta-analysis confirms that proteinuria strongly predicts coronary heart disease}

Markers of kidney dysfunction have been associated with an increased risk of coronary heart disease (CHD), although this association has not been characterized in depth. Perkovic and colleagues, therefore, performed a metaanalysis in order to estimate the strength of the relationship between levels of protein or albumin in the urine and risk of CHD.

An electronic literature search identified 26 cohort studies, including 169,949 individuals, that reported the risk of $\mathrm{CHD}$ associated with the following: proteinuria (total urinary protein $>300 \mathrm{mg} /$ day, $n=10$ ), macroalbuminuria (urinary albumin $>300 \mathrm{mg} /$ day, $n=9$ ), microalbuminuria (urinary albumin $30-300 \mathrm{mg} /$ day, $n=7$ ), and any level of albuminuria $(n=6)$. A total of 7,117 coronary events ( $27 \%$ fatal) were reported.

Compared with people who had normal levels of protein in the urine, individuals with proteinuria were $50 \%$ more likely to experience CHD (over a mean follow-up period of 4-27 years). The strength of this association was consistent across subgroups of diabetes status and ethnicity, among others. Individuals with microalbuminuria were $50 \%$ more likely to develop CHD than those without, while the risk of CHD was more than doubled for those with macroalbuminuria. The levels of urinary protein were, however, generally determined by means of dipstick testing, which has a low sensitivity and specificity, while albuminuria was detected by use of laboratory analysis.

Perkovic et al. were unable to determine whether proteinuria has a direct role in the development of CHD, but they recommend evaluating proteinuria in the assessment of $\mathrm{CHD}$ risk.

Original article Perkovic V et al. (2008) The relationship between proteinuria and coronary risk: a systematic review and meta-analysis. PLoS Med 5: e207

\section{Laparoscopic nephrectomy can cause testicular pain}

Testicular pain has been reported as a potential adverse effect of laparoscopic renal surgery, but is generally only identified after direct questioning. Gjertson and Sundaram, therefore, prospectively studied male patients scheduled for laparoscopic kidney and adrenal surgery to determine the incidence of testicular pain postsurgery.

The researchers assessed 64 men (mean age 50 years) in which a total of 68 procedures (60 unilateral, 4 bilateral) were carried out. Initial postoperative physical examination revealed no changes from baseline, and no patient described testicular pain. After discharge from the hospital, 14 patients developed testicular pain (4-7 days after surgery), which was ipsilateral to the surgical side in 13 cases. All patients reporting pain underwent either donor nephrectomy or radical nephrectomy. These men were a mean 10 years younger, had an increased intraoperative urine output, and were less likely to have a history of surgery, than those who experienced no pain. However, the authors were unsure whether these factors were linked to testicular pain or reflected the limited cohort size. Instead, they explained that ligation of the gonadal vein seemed to be associated with the risk of pain: testicular pain developed after 13 (33\%) of the 39 procedures where the gonadal vein was ligated, compared with just 1 case when the gonadal vein was preserved $(3.4 \%$ of 29 procedures).

The authors suggest that patients undergoing donor or radical nephrectomy should be informed of the potential for testicular pain, and that preservation of the gonadal vein might confer a protective effect.

Original article Gjertson CK and Sundaram CP (2008) Testicular pain following laparoscopic renal surgery. J Urol 180: 2037-2040

\section{Percutaneous nephrolithotomy is 'safe and effective' in transplanted kidneys}

Despite case reports of successful percutaneous nephrolithotomy in kidney transplants, concerns persist about the risks of technical difficulties, injury of adjacent organs, and impaired 